\title{
Saúde e trabalho de motoristas de transporte alternativo
}

\author{
Health and work of alternative transportation drivers \\ Salud y trabajo de conductors de transporte alternativo
}

Adriana Brandão Ribeiro ${ }^{1}$, Fernanda Matos Fernandes Castelo Branco ${ }^{1 *}$, Tancredo Castelo Branco Neto'.

\section{RESUMO}

Objetivo: caracterizar as condições de saúde e trabalho dos motoristas de transporte alternativo. Métodos: estudo descritivo, realizado com 40 motoristas no município de Oiapoque, no período de fevereiro e março de 2018. Utilizou-se questionário fechado que aborda aspectos da percepção dos motoristas sobre a sua saúde e trabalho. Resultados:42,5\% apresentam estresse, 52,5\% não tem problema de postura e coluna, $52,5 \%$ às vezes sentem dores de cabeça, $90 \%$ tem resfriado e gripe, $70,3 \%$ apresentam tosse seca, $55,3 \%$ tem dor ou ardor na garganta, $62,5 \%$ às vezes se sentem nervosos, $65 \%$ são depressivos, $50 \%$ tem angústia, 52,5\% sentem medo, 52,5\% tem ansiedade, $73 \%$ tem insônia, 57,5\% tem cansaço e sono, 56,4\% apresentam algum grau de obesidade, 7,7\% possuem problemas de coração (hipertensão e palpitações), 2,6\% tem diabetes. Conclusão: A partir dos resultados sobreleva a importância de discussões reflexivas acerca da vulnerabilidade, bem como a necessidade de mais estudos específicos com esta clientela a fim de nortear estratégias educativas voltadas a esta classe trabalhista, considerando os aspectos de saúde e trabalho.

Palavras-chave: Condução de veículos, Condições de trabalho, Saúde na Fronteira.

\begin{abstract}
Objective: to characterize the health and work conditions of alternative transportation drivers. Methods: A descriptive study was carried out with 40 drivers in the city of Oiapoque, in the period of February and March of 2018. A closed questionnaire was used that addresses aspects of the drivers' perception of their health and work. Results: $42.5 \%$ have stress, $52.5 \%$ do not have a posture and spine problem, $52.5 \%$ sometimes feel headaches, $90 \%$ have a cold and the flu, $70.3 \%$ have dry cough, $55.3 \%$ have $62.5 \%$ sometimes feel nervous, $65 \%$ are depressed, $50 \%$ have anxiety, $52.5 \%$ feel fear, $52.5 \%$ have anxiety, $73 \%$ have insomnia, $57.5 \%$ have anxiety, fatigue and sleep, $56.4 \%$ have some degree of obesity, $7.7 \%$ have heart problems (hypertension and palpitations), $2.6 \%$ have diabetes. Conclusion: Based on the results, the importance of reflective discussions about vulnerability is emphasized, as well as the need for more specific studies with this clientele in order to guide educational strategies geared to this class, considering the health and work aspects.
\end{abstract}

Keywords: Vehicle driving, Work conditions, Health at the Border.

\section{RESUMEN}

Objetivo: caracterizar las condiciones de salud y trabajo de los conductores de transporte alternativo. Métodos: se realizó un estudio descriptivo con 40 conductores en la ciudad de Oiapoque, en los meses de febrero y marzo de 2018. Se utilizó un cuestionario cerrado que aborda aspectos de la percepción de los

${ }^{1}$ Universidade Federal do Amapá (UNIFAP), Oiapoque-Amapá *E-mail: fmfernandescb@gmail.com 
conductores sobre su salud y trabajo. Resultados: El $42.5 \%$ tiene estrés, el $52.5 \%$ no tiene problemas de postura y columna vertebral, el $52.5 \%$ a veces siente dolores de cabeza, el $90 \%$ tiene un resfriado y la gripe, el $70.3 \%$ tiene tos seca, el $55.3 \%$ tiene El $62.5 \%$ a veces se siente nervioso, el $65 \%$ está deprimido, el $50 \%$ tiene ansiedad, el $52.5 \%$ siente miedo, el $52.5 \%$ tiene ansiedad, el $73 \%$ tiene insomnio, el $57.5 \%$ tiene ansiedad, fatiga y sueño, el 56,4\% tiene algún grado de obesidad, el 7,7\% tiene problemas cardíacos (hipertensión y palpitaciones), el 2,6\% tiene diabetes.Conclusión: Sobre la base de los resultados, se enfatiza la importancia de las discusiones reflexivas sobre la vulnerabilidad, así como la necesidad de estudios más específicos con esta clientela para orientar las estrategias educativas orientadas a esta clase, considerando los aspectos de salud y trabajo.

Palabras clave: Conducción de vehículos, Condiciones de trabajo, Salud en la frontera.

\section{INTRODUÇÃO}

Os motoristas, pertencem a uma profissão que exige muita atenção, precisão na realização das ações, autocontrole, reflexo imediato, análise e interpretação das informações fornecidas pelos equipamentos dos veículos (COSTA LB, et al., 2006).

Desta via, o trabalho de motorista pode levar danos à saúde; seu cotidiano contribui para hábitos de vida pouco saudáveis, para o sedentarismo, práticas alimentares inadequadas, resultando-se sobrepeso, uso de álcool e tabaco, entre outros problemas. Além desses, a profissão acarreta transtornos na convivência familiar e na vida social (MASSON VA e MONTEIRO MI, 2010).

Uma pesquisa revelou que, em relação a autopercepção de saúde de motoristas, $14,7 \%$ dos entrevistados relataram sua saúde como regular/ruim; mais de $20 \%$ dos entrevistados admitiram fumar naquele momento; $40 \%$ não praticavam o mínimo de atividade física recomendada para promoção da saúde; e 3/4 foram classificados nas categorias de sobrepeso/obesidade pelo Índice de Massa Corporal (IMC). As maiores prevalências de dores se referiam a região lombar, torácica, pescoço e ombros, e $9 \%$ dos entrevistados apresentaram Transtornos Psiquiátricos Menores (TPM). Portanto, os motoristas representam prevalências preocupantes de fatores de risco para doenças crônicas (MOURA NETO AB e SILVA MC, 2012).

Estudo realizado com motoristas de transporte de cargas, se aprofundou quanto a essa categoria de profissionais; foram investigadas as condições de vida, trabalho e saúde dos motoristas e entrevistados 185 trabalhadores; os dados em relação à saúde foram que 33 deles - ou seja, 17,84\% - eram hipertensos; 18 participantes - 9,73\% - eram diabéticos; 38 deles - 20,54\%, - apresentavam dislipidemia; e 55 - 29,73\% faziam uso de medicação controlada (RAMOS BH, et al., 2018)

Nesse sentido e embasado no que foi descrito acima faz-se necessário destacar o meio de transporte alternativo no município de Oiapoque, popularmente conhecido como "pirata", é feito por carros tracionados "pickup".

O transporte alternativo "pirata" vem sendo utilizado há vários anos pela população no município de Oiapoque pelo fato de oferecer praticidade e rapidez, principalmente nos períodos de difícil tráfego na BR 156, tal como no inverno, devido aos $110 \mathrm{Km}$ existentes sem asfalto, sendo muitas vezes, o único meio de transporte viável.

Considerando-se que a atividade de trabalho dos motoristas de transporte alternativo no município de Oiapoque tem grande importância para a população local, tendo-se em vista as condições de trabalho, os riscos a que são expostos e a grande responsabilidade de sua atividade durante o transporte cotidiano de passageiros, além da falta de pesquisas nesta população específica da fronteira franco brasileira - o que por si só justifica a realização deste estudo -, surgiu o seguinte questionamento: Quais são as condições de saúde e trabalho dos motoristas de transporte alternativo em Oiapoque? Deste modo, este estudo objetivou caracterizar as condições de saúde e trabalho dos motoristas de transporte alternativo. 


\section{MÉTODOS}

Estudo descritivo com abordagem quantitativa. Participaram deste estudo 40 motoristas, tendo como critério de inclusão: trabalhar há mais de um ano com transporte alternativo, sendo excluídos os motoristas que apresentaram sinais ou sintomas clínicos sugestivos de embriaguez/intoxicação devido ao uso de substâncias psicoativas, bem como os que não aceitaram participar da pesquisa.

Durante a execução da pesquisa, esta classe trabalhista não contava com nenhuma entidade, associação ou órgão competente que os represente, portanto, não podendo estimar a quantidade exata de pessoas de tal profissão em Oiapoque; a abordagem ocorreu de forma direta e individualizada com os participantes do estudo. É válido lembrar que os motoristas não foram submetidos a exames clínicos ou consultas médicas, portanto os agravos de saúde e queixas clínicas forma baseadas em auto relatos dos entrevistados.

A pesquisa envolveu aplicação de questionários. Para caracterização sociodemográfica e laboral dos participantes do estudo, foi utilizado um questionário com questões fechadas, elaborado pelos autores deste estudo. E na identificação das condições de saúde e trabalho o instrumento da pesquisa foi baseado e adaptado de estudo previamente realizado, sendo selecionado parte do questionário que continha os fatores de interesse para esta pesquisa (PENTEADO RZ, et al., 2008).

O questionário foi aplicado de forma individual, após leitura e assinatura do Termo de Consentimento Livre e Esclarecido (TCLE), em um local reservado, para que o participante se sentisse seguro a colaborar com a pesquisa. Foi utilizada uma abordagem cautelosa e que garantisse maior privacidade nas respostas; caso o entrevistado não se sentisse à vontade para prosseguir com o estudo, este era interrompido. Além disso, foi assegurado o direito a ressarcimento ou indenização no caso de quaisquer danos eventualmente produzidos pela pesquisa.

A coleta somente ocorreu após realizados de todos os trâmites legais e necessários para sua execução que ocorreu nos meses de fevereiro e março de 2018. Os questionários foram aplicados durante o período diurno, com duração aproximada de 20 minutos, nos locais de pontos localizados à beira rio do município de Oiapoque, no qual ocorre a maior parte dos embarques e desembarques de passageiros.

O trabalho foi submetido à Plataforma Brasil e encaminhado ao Comitê de Ética e Pesquisa da Universidade Federal do Amapá (UNIFAP) para apreciação, aprovado com CAAE: 80769817.0.0000.0003. Os dados foram analisados de forma descritiva, através de tabelas de frequência.

\section{RESULTADOS}

Dos participantes entrevistados, $42,5 \%$ tinham até 29 anos; $35 \%$ eram naturais do Pará; $35 \%$ contavam de 4 a 7 anos de profissão; $65 \%$ relataram que as questões financeiras os levaram a exercer a profissão; $52,5 \%$ tinham ensino fundamental; $60 \%$ eram solteiros; $87,5 \%$ residiam em Oiapoque; $40 \%$ residiam com três pessoas; $100 \%$ eram brasileiros; $42 \%$ trabalhavam de $14 \mathrm{~h}$ às $16 \mathrm{~h} /$ dia; $90 \%$ possuíam carro próprio; $40 \%$ faziam três viagens semanalmente; $57,5 \%$ dormiam de 08 a 09 horas por dia; $75 \%$ tinham preferência por viajar no período da noite.

Este estudo evidenciou que $40 \%$ dos participantes fazem três viagens por semana, e o trajeto percorrido por esses motoristas é aproximadamente de $600 \mathrm{~km}$; levando-se em consideração o embarque e desembarque de passageiros e local de entrega de carga, esse trajeto percorrido pode até duplicar. Muitas vezes essas viagens chegam a ser de Oiapoque a Macapá ida e volta, ou seja, os motoristas não descansam no período, o que os leva a um grande desgaste físico e mental.

Em relação aos hábitos de consumo de comportamentos dos entrevistados, $67,5 \%$ "sempre" viajam com o ar condicionado ligado; $80 \%$ "sempre" consomem comidas gordurosas, apimentadas e frituras; $67,5 \%$ bebem café puro com frequência; $62,5 \%$ bebem 8 ou mais copos de água por dia; $57,5 \%$ "nunca" viajam com os vidros da cabine abertos; $45 \%$ não têm o hábito de fumar; $50 \%$ não fazem uso de rebite; $92,5 \%$ não 
fazem uso de outras drogas (cocaína, maconha, crack, entre outras.), $65 \%$ "às vezes" fazem uso de bebida alcoólica; $52,5 \%$ "às vezes" tomam energéticos, $62,5 \%$ "às vezes" fazem uso de pastilhas para a garganta, dados estes evidenciados na Tabela 1.

Tabela 1 - Frequência de hábitos de consumo e comportamento referidos pelos motoristas, $n=40$. Oiapoque-AP, 2018.

\begin{tabular}{lrrr}
\hline Hábitos & Sempre (\%) & Nunca (\%) & Às vezes (\%) \\
\hline Viaja sempre com vidros da Cabine Abertos & 10,0 & 57,5 & 32,5 \\
Viaja com ar condicionado ligado & 67,5 & 0,0 & 32,5 \\
Fuma & 30,0 & 45,0 & 25,0 \\
Toma Bebida alcóolica & 22,5 & 12,5 & 65,0 \\
Toma Energéticos & 27,5 & 20,0 & 52,5 \\
Toma rebite & 15,0 & 50,0 & 35,0 \\
Usa drogas (cocaína, maconha, crack e outros) & 0,0 & 92,5 & 7,5 \\
Chupa Pastilha para a garganta & 17,5 & 20,0 & 62,5 \\
Come alimentos gordurosos, frituras ou apimentados & 80,0 & 0,0 & 20,0 \\
Toma café puro & 67,5 & 7,5 & 25,0 \\
\hline & Às vezes & $\mathbf{1 ~ a ~ 7 ~ c o p o s ~ p o r ~}$ & $\mathbf{8}$ copos ou \\
Bebe água & \multicolumn{2}{c}{ dia } & mais por dia \\
\hline
\end{tabular}

Fontes: Dados da pesquisa (2018)

Em relação aos problemas de saúde, pode-se visualizar as várias queixas na Tabela 2, sendo as principais delas: $52,5 \%$ as vezes sentem dor de cabeça, $61,5 \%$ as vezes apresentam cãibras, tensão e/ou dor na nuca e/ou dor nos ombros, braços e mãos, $65,0 \%$ as vezes sentem cãibras, tensão e/ou dor na nuca e/ou no pescoço, $75 \%$ nunca rangem os dentes, $60,0 \%$ nunca ter dor na ATM, $52,5 \%$ as vezes apresentam problemas de postura, de coluna e lou dor nas costas, $85,0 \%$ nunca tiveram perda da audição ou dificuldade para entender conversação ao telefone ou em ambiente de festas e com ruído, 70,0\% nunca apresentaram apito ou zumbido no ouvido, $62.5 \%$ nunca tiveram otite, inflamação ou dor de ouvido, 92,5\% nunca apresentaram tonturas, náuseas e vômitos, $90,0 \%$ as vezes apresentam resfriados e gripes, $75,0 \%$ as vezes apresentam nariz ressecado ou escorrendo, $52,5 \%$ as vezes tem rinite, $77,5 \%$ tem sinusite, $72,5 \%$ as vezes tem bronquite, $95,0 \%$ as vezes apresentam asma e azia, "queimação" no estômago, gastrite ou refluxo, $42,5 \%$ sempre apresentam sentimentos negativos de estresse, $62,5 \%$ as vezes se sentem nervosos, $65 \%$ as vezes sentem depressão, $47,5 \%$ nunca sentem insegurança, $50 \%$ as vezes sentem angústia, $52,5 \%$ as vezes apresentam medo e ansiedade, $60 \%$ as vezes apresentam machucados, placas brancas ou feridas na boca, $73 \%$ nunca apresentam insônia, $57,5 \%$ as vezes cansaço e sono, $75 \%$ as vezes ronco, 56,4\% apresentam obesidade, 7,7\% problemas do coração, hipertensão, taquicardia, 2,6\% palpitações, 2,6\% diabetes e 46,2\% problemas de circulação, varizes ou peso e cansaço nas pernas.

No que concerne à frequências de queixas de saúde relacionada à voz, destacaram-se os seguintes dados: dos entrevistados $52,6 \%$ "nunca" tem pigarro, $87,2 \%$ não tem perda da voz, $79,4 \%$ não tem cansaço o falar, $94,7 \%$ não tem falha ou quebra da voz ao falar, $91,7 \%$ não desconforto ou dor ao falar, $70,3 \%$ não tem sensação de "bolo" ou dificuldade para engolir, $91,9 \%$ não tem falha da voz ao cantar, $83,3 \%$ não senti desconforto ao cantar, $70,3 \%$ "às vezes" tem tosse seca, $57,9 \%$ "às vezes" sentem a garganta seca e coceira, $55,3 \%$ "às vezes" tem dor ou ardor a garganta. 
Tabela 2 - Frequência de problemas de saúde referidos pelos motoristas, n=40. Oiapoque-AP, 2018.

\begin{tabular}{|c|c|c|c|}
\hline Variáveis Queixas & Sempre (\%) & Nunca (\%) & Às vezes (\%) \\
\hline Dor de cabeça & 12,5 & 35,0 & 52,5 \\
\hline Cãibras, tensão e/ou dor nos ombros, braços e mãos & 20,5 & 17,9 & 61,5 \\
\hline Cãibras, tensão e/ou dor na nuca e/ou no pescoço & 17,5 & 17,5 & 65,0 \\
\hline Range os dentes & 0,0 & 75,0 & 25,0 \\
\hline Dor na ATM (articulação têmporo-mandibular) & 10,0 & 60,0 & 30,0 \\
\hline Problemas de postura, de coluna e /ou dor nas costas & 42,5 & 5,0 & 52,5 \\
\hline $\begin{array}{l}\text { Perda da audição ou dificuldade para entender } \\
\text { conversação ao telefone ou em ambiente de festas e } \\
\text { com ruído }\end{array}$ & 7,5 & 85,0 & 7,5 \\
\hline Apito ou zumbido no ouvido & 0,0 & 70,0 & 30,0 \\
\hline Otite, inflamação ou dor de ouvido & 5,0 & 62,5 & 32,5 \\
\hline Tonturas, náuseas e vômitos & 0,0 & 92,5 & 7,5 \\
\hline Resfriados e gripes & 5,0 & 5,0 & 90,0 \\
\hline Nariz ressecado ou escorrendo & 2,5 & 22,5 & 75,0 \\
\hline Rinite & 5,0 & 52,5 & 42,5 \\
\hline Sinusite & 12,5 & 77,5 & 10,0 \\
\hline Bronquite & 5,0 & 72,5 & 22,5 \\
\hline Asma & 0,0 & 95,0 & 5,0 \\
\hline Azia, "queimação" no estômago, gastrite ou refluxo & 2,5 & 95,0 & 2,5 \\
\hline Sentimentos negativos de estresse & 42,5 & 17,5 & 40,0 \\
\hline Nervoso (a) & 20,0 & 17,5 & 62,5 \\
\hline Depressão & 5,0 & 30,0 & 65,0 \\
\hline Insegurança & 7,5 & 47,5 & 45,0 \\
\hline Angústia & 10,0 & 40,0 & 50,0 \\
\hline Medo & 2,5 & 45,0 & 52,5 \\
\hline Ansiedade & 0,0 & 47,5 & 52,5 \\
\hline $\begin{array}{l}\text { Machucados, placas brancas ou feridas na boca } \\
\text { (lábios, língua, bochechas ou céu da boca) }\end{array}$ & 27,5 & 12,5 & 60,0 \\
\hline Insônia (falta de sono e problemas para dormir) & 0,0 & 73,0 & 27,0 \\
\hline Cansaço e sono & 10,0 & 32,5 & 57,5 \\
\hline \multirow[t]{2}{*}{ Ronco } & 25,0 & 0,0 & 75,0 \\
\hline & & $\mathbf{n}$ & $\%$ \\
\hline \multirow{2}{*}{\multicolumn{2}{|c|}{$\begin{array}{l}\text { Problemas dentários } \\
\text { Variáveis Queixas }\end{array}$}} & 25 & 64,1 \\
\hline & & $\mathbf{n}$ & $\%$ \\
\hline \multicolumn{2}{|l|}{ Obesidade } & 22 & $\overline{56,4}$ \\
\hline \multicolumn{2}{|l|}{ Problemas de coração, hipertensão, taquicardia } & 3 & 7,7 \\
\hline \multicolumn{2}{|l|}{ Palpitações } & 1 & 2,6 \\
\hline \multicolumn{2}{|l|}{ Diabetes } & 1 & 2,6 \\
\hline \multicolumn{2}{|c|}{ Problemas de circulacão, varizes ou peso e cansaco nas pernas } & 18 & 46,2 \\
\hline
\end{tabular}

Fontes: Dados da pesquisa (2018).

$\mathrm{Na}$ Tabela 3 foi evidenciado que os fatores que mais afetam a voz a e garganta foram: falar muito (65\%), falar alto $(65 \%)$, poeira $(77,5 \%)$, fumaça $(65 \%)$, vento, friagem ou mudança de clima e temperatura $(75 \%)$, ar condicionado (65\%). Foi evidenciado ainda que apenas $22,5 \%$ dos motoristas entrevistados conhecem 0 trabalho de fonoaudiologia. 
Tabela 3 - Frequência do que afeta a voz e a garganta relatado pelos motoristas, n=40. Oiapoque-AP, 2018.

\begin{tabular}{lr}
\hline Variáveis & $\%$ \\
\hline Falar Muito & 65,0 \\
Falar Alto & 65,0 \\
Gritar & 57,5 \\
Cantar & 15,0 \\
Poeira & 77,5 \\
Fumaça & 65,0 \\
Vento, friagem ou mudança de clima e temperatura & 75,0 \\
Ar condicionado & 65,0 \\
Fumo & 32,5 \\
Alimentos gelados e bebidas geladas & 10,0 \\
Álcool & 5,0 \\
Drogas & 2,5 \\
Cheiros fortes e produtos químicos & 7,5 \\
Frituras, alimentos apimentados, pesados e gordurosos & 15,0 \\
Estresse, nervoso, ansiedade ou emoções fortes & 2,5 \\
Café & 7,5 \\
Refrigerantes e bebidas energéticas com gás & 7,5 \\
\hline Font Dados da pesquisa (2018)
\end{tabular}

Fontes: Dados da pesquisa (2018).

\section{DISCUSSÃO}

Há necessidade de se preocupar com as horas dormidas dos motoristas; o estudo em questão mostra que $57,5 \%$ dos motoristas dormem em média de 08 a 09 horas diariamente e que $75 \%$ dos participantes têm preferência por viagens noturnas. O que contradiz estudo realizado por Wendler E et al. (2003) com motoristas que fazem longos trajetos por exemplo, mostraram que mais da metade dos participantes dormiam de 3 a 4 horas por dia; muitos se encontravam sob ação dos psicoestimulantes e passavam mais de 2 dias sem dormir.

O resultado da investigação de Nascimento EC et al. (2007) aproxima-se do presente estudo: os participantes relataram que dormiam de 04 a 06 horas por dia, porém mostrou significância estatística entre as horas de descanso e os acidentes que ocorriam nas estradas, ou seja, revelou que quanto menos os motoristas dormem, mais eles correm o risco de ocasionarem acidentes.

Fator que merece ser discutido são as práticas alimentares dos motoristas participantes que podem trazer prejuízo à saúde, como afirma Lago M (2009); que relata uma elevada frequência de fatores de risco cardiovasculares em motoristas, possível reflexo de má alimentação, falta de condicionamento físico e nível de estresse elevado. Os caminhoneiros de rota longa se alimentam em restaurantes à beira das estradas, com oferta de alimentos de alto valor calórico e baixo valor nutritivo, assim como calorias vazias, vindas especialmente pelo uso de álcool. Má digestão e sensação desagradável no estômago são sintomas comuns aos caminhoneiros (CAVAGIONIO LC, et al., 2009). Os motoristas aqui analisados também fazem a maioria de suas refeições em restaurantes ou lanchonetes em beira de estrada. O estudo mostrou que 2,5\% dos participantes queixam-se de azia, "queimação" no estômago, gastrite ou refluxo, o que pode ser um reflexo de uma prática alimentar inadequada.

Em relação à obesidade e ao sedentarismo, 56,4\% dos participantes referiram algum grau de obesidade, o que pode ser resultado de práticas alimentares inadequadas e falta de tempo para praticar algum tipo de exercício físico. Na pesquisa de Coser J et al. (2009), 42\% dos caminhoneiros apresentaram obesidade, $44 \%$, sobrepeso, e $78 \%$, obesidade visceral. Estudos realizados anteriormente identificaram que a falta de tempo para realizar uma alimentação equilibrada resulta em distúrbios gastrintestinais, sintomas e doenças relacionadas ao sistema musculoesquelético como a principal causa de morbidade em motoristas (SIVIERO $A B$, et al., 2005). Uma alimentação à base de alimentos gordurosos e com excesso de sal, além de resultar em obesidade, confere risco à saúde por estar relacionada a quadros hipertensivos e doenças cardíacas (NERI M, et al., 2005). 
Além de todas essas condições já descritas, os motoristas estão expostos a grandes poluições, tal como fumaças, poeiras ou friagens no decorrer das viagens. Este estudo detecta que a voz, juntamente com o sistema respiratório dos motoristas de transporte alternativo, também está exposta à poluição, a hábitos e costumes que acometem este sistema, por exemplo, falar muito e falar alto, citados na pesquisa. Portanto pesquisa realizada previamente demonstrou que $57,5 \%$ dos motoristas "nunca" viajam com os vidros da cabine abertos e $67,5 \%$ "sempre" viajam com o ar condicionado ligado. Resultados contrários forma encontrados em outras investigações já realizadas- $52,5 \%$ sempre viajam com os vidros da cabine abertos e $45,5 \%$ não viajam com o ar condicionado ligado, o que, segundo eles, expõe o indivíduo ao vento e à friagem, à poeira, à fumaça, a queimadas, poluição em geral, fatores químicos que causam prejuízos ambientais que acometem o sistema respiratório, ocasionando algumas doenças ocupacionais (MIRANDA JS, et al., (2005)

Em relação ao hábito de fumar, o estudo mostrou um resultado surpreendente quando comparado a outros estudos: $45 \%$ dos participantes não fumam atualmente. Um resultado bastante satisfatório para esta categoria dos motoristas. No entanto, $30 \%$ dos participantes "sempre" e $25 \%$ "às vezes" são fumantes, ainda assim um percentual muito alto comparado a outros estudos como, por exemplo, que encontraram prevalência de $21,25 \%$ de usuários regulares "sempre" e $11,25 \%$ de usuários irregulares "às vezes" (PENTEADO RZ, et al., 2008). Outra pesquisa corroborou com esse achado, no qual foi observado que $21 \%$ dos motoristas eram tabagistas, ou seja, eles consumiam em média 17 cigarros ao dia (MASSON VA e MONTEIRO MI, 2010). O uso de álcool foi uma variável presente nesse estudo fato este concordante com investigação que mostrou que consumo de bebidas alcoólicas foi relatado por $49,4 \%$ dos caminhoneiros pesquisados (GUEDES HM, et al., 2010)

Os pesquisadores Nascimento EC et al. (2007); observaram em sua pesquisa que $91 \%$ dos caminhoneiros tinham o hábito de ingerir bebidas alcoólicas durantes as viagens de trabalho; desses, $24 \%$ usavam álcool todos os dias e $35 \%$ bebiam de duas a três vezes por semana. Foi discutido ainda que os motoristas que consomem bebidas alcoólicas durante as jornadas de trabalho podem se envolver em acidentes nas estradas, portanto 14 caminhoneiros que haviam se envolvido em acidentes, 11, ou seja, $79 \%$, faziam uso de álcool em postos de combustíveis.

Dos motoristas entrevistados, $52,5 \%$ relataram usar "às vezes" algum tipo de energético, com o objetivo de aliviar o sono e o cansaço físico. No estudo de Penteado RZ et al. (2008); o resultado foi bem diferente entre motoristas de caminhão; conforme seus dados, $80,5 \%$ "nunca" fazem uso de energéticos. Nesse presente estudo, o uso de substâncias psicoativas - como o rebite, a cocaína, a maconha, o crack e outros foram relatados; são utilizadas no intuito de reduzir o sono e diminuir o cansaço; em Oiapoque, 15\% dos participantes admitiram fazer uso "sempre", e 35\%, eventualmente, "às vezes", da substância conhecida por eles como rebite. Poucos têm conhecimento de que se trata de Anfetamina, um estimulante do sistema nervoso central que pode levar a graves problemas de saúde. Em relação a outras substâncias, 7,5\% admitiram fazer uso eventualmente.

É comum o uso do rebite entre motoristas (17\%), além de outras drogas, em menor escala: cocaína $12 \%$; "bolinha" (estimulantes, geralmente anfetaminas) $3 \%$; calmantes $5 \%$; cola $4 \%$; LSD $3 \%$; e crack $1,5 \%$ (VILARINHO B, et al., 2002). Outra pesquisa evidenciou que $66 \%$ dos caminhoneiros de estrada costumavam usar anfetaminas durante o percurso de viagem, dentre os quais $27 \%$ as usavam diariamente e $60 \%$ de duas a três vezes por semana (NASCIMENTO EC, et al., 2007).

Um índice ainda mais alto, 96,8\% dos entrevistados confirmaram o uso pelo menos uma vez ou rotineiramente, com a finalidade de aumentar o tempo de vigília; e afirmaram que a compra dessas drogas ocorria em postos de gasolina, borracharia, farmácia, restaurantes, lanchonete, mercadinho e em outro país de área de estudo investigada (WENDLER E, et al., 2003). Sob uma visão ampla da área da enfermagem, há uma necessidade de realizar ações educativas, de promoção e prevenção, para tais profissionais, bem como trabalhar em conjunto com os órgãos fiscalizadores nas estradas, visando a um controle de motoristas que dirigem sob efeito de álcool e outras drogas. Esse trabalho deve ser intenso, repleto de informações e conscientização pela melhoria da qualidade de vida desses profissionais.

$\overline{\text { REAS/EJCH | Vol. 11(13) | e1189 | DOI: https://doi.org/10.25248/reas.e1189.2019 Página } 7 \text { de } 8}$ 
Por fim, devido à inexistência de dados acerca desta população investigada, ou seja, dos motoristas de transporte alternativo do município do Oiapoque, no estado do Amapá, foi possível realizar um panorama geral acerca de suas condições de saúde e trabalho. A partir da análise destas variáveis, se pôde avaliar que essa categoria de trabalhadores está vulnerável e predisposta a desenvolver enfermidades, sendo esta a maior potencialidade deste estudo. Além do que os dados existentes na literatura são voltados a motoristas de caminhão e transporte de carga, sendo os de transporte alternativo inexistentes na área.

Entretanto devido ao número limitado da amostra, os dados não podem ser generalizados. Portanto, outros estudos se fazem pertinentes a fim de investigar com mais rigor as condições e da organização do trabalho de motoristas de transporte alternativo e de como elas interferem nos espaços de vida familiar, social e de trabalho. Sob outro ponto de vista, saber mais a respeito das percepções, receios, satisfações e expectativas sobre as relações entre saúde, trabalho e qualidade de vida e também sobre ações possíveis e futuras a serem instituídas para a transformação das condições de trabalho e a melhoria da atividade profissional.

\section{CONCLUSÃO}

A partir das variáveis analisadas se pôde avaliar que essa categoria de trabalhadores está vulnerável e predisposta a desenvolver enfermidades, como: doenças crônicas (hipertensão e diabetes), colesterol alto, dores por problemas de coluna, obesidade, estresse, depressão, ansiedade, problemas respiratórios e gastrointestinais, causadas pelo trabalho rotineiro de dirigir longos trajetos e pela impossibilidade de se levar uma vida com hábitos saudáveis por falta de tempo e por viver em constante atividade. A partir dos resultados sobreleva a importância de discussões reflexivas acerca da vulnerabilidade, bem como a necessidade de mais estudos específicos com esta clientela a fim de nortear estratégias educativas voltadas a esta classe trabalhista, considerando os aspectos de saúde e trabalho.

\section{REFERÊNCIAS}

1. COSTA LB, et al. Morbidade declarada e condições de trabalho: o caso dos motoristas de São Paulo e Belo Horizonte. São Paulo em Perspectivas, 2006;17 (2):54-67.

2. MASSON VA, MONTEIRO MI. Estilo de vida, aspectos de saúde e trabalho de motoristas de caminhão. Revista Brasileira de Enfermagem, 2010; 63(4):533-540.

3. PENTEADO RZ, et al. Trabalho e saúde em motoristas de caminhão no interior de São Paulo. Saúde Sociedade, 2008;17(4): 35-45.

4. MOURA NETO AB, SILVA MC. Diagnóstico das condições de trabalho, saúde e indicadores do estilo de vida de trabalhadores do transporte coletivo da cidade de Pelotas - RS. Revista Brasileira de Atividade Física e Saúde, Pelotas/RS, 2012; 17(5): 347-358.

5. RAMOS BH, et al. Condições de vida, trabalho e saúde de motoristas de transporte de cargas. Revista enfermagem UFPE online, 2018; 12(1):150-159.

6. WENDLER E, et al. Uso de anfetaminas por motoristas de caminhão para reduzir o sono. Publ UEPG Ciências Biológicas e da Saúde, 2003; 9(3): 7-14.

7. NASCIMENTO EC, et al. Uso de álcool e anfetaminas entre caminhoneiros de estrada. Revista Saúde Pública, 2007; 41(2): 290-293.

8. LAGO M. Perfil lipídico e fatores de risco cardiovasculares em motoristas de caminhão da região norte do estado do Rio Grande do Sul. Monografia. Carazinho: Universidade Luterana do Brasil; 2009.

9. CAVAGIONI LC, et al. Agravos à saúde, hipertensão arterial e predisposição ao estresse em motoristas de caminhão. Revista da Escola. Enfermagem. USP, 2009;43 (spe2): 1267-1271.

10. COSER J, et al. Triagem dos perfis lipídico e glicídico em caminhoneiros que trafegam no centro unificado de fronteira, entre Brasil e Argentina. Revista Brasileira. Análises.clínicas, 2009; 41(3):223-228.

11. SIVIERO AB, et al. Prevalência de perda auditiva em motoristas de ônibus do transporte coletivo da cidade de MaringáPR. Revista Cefac, 2005; 7(3): 376-381.

12. NERI M, et al. Condições de saúde no setor de transporte rodoviário de cargas e de passageiros: um estudo baseado na Pesquisa Nacional por Amostra de Domicílios. Caderno de Saúde Pública, 2005; 21(4): 1107-1123.

13. MIRANDA JS, et al. Percepções sobre a voz/saúde vocal por motoristas de caminhão. Revista da Sociedade Brasileira de Fonoaudiologia, 2005; (supl esp):170p.

14. GUEDES HM, et al. Fatores de risco para o desenvolvimento de hipertensão arterial entre motoristas caminhoneiros. Cogitare Enfermagem, 2010; 15(4): 652-658.

15. VILARINHO B, et al. Caminhoneiros de rota curta e sua vulnerabilidade ao HIV, Santos, SP. Revista Saúde Pública, 2002;36(suppl 4): 61-67. 